\title{
Study of Error Analysis and Sources of Uncertainty in the Measurement of Residual Stresses by the X-Ray Diffraction
}

\author{
E.T. Carvalho Filho ${ }^{1, a, *}$, J.T.N. Medeiros ${ }^{1, b}$, L.G. Martinez ${ }^{2, c}$ and V.C. Pinto ${ }^{1, d}$ \\ ${ }^{1}$ Federal University of Rio Grande do Norte, Brazil \\ ${ }^{2}$ Nuclear and Energy Research Institute, Brazil \\ ${ }^{3}$ Federal Institute of Rio Grande do Norte, Brazil

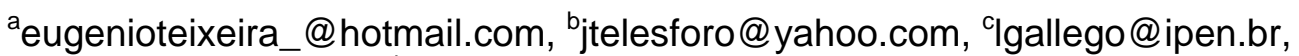 \\ dvinicius_c_pinto@hotmail.com
}

Keywords: Residual Stress, X Ray Diffraction, Repeatability, Reproducibility

\begin{abstract}
The aim of this work is to analyze the sources of errors inherent to the residual stress measurement process by X-ray diffraction technique making an interlaboratory comparison to verify the reproducibility of the measurements. For this work were machined specimens with grinding finish, with polishing finish and to be a reference standard an iron powder was used To verify the deviations caused by the equipment, those specimens were positioned and with the same analysis condition, seven measurements were carried. To verify sample positioning errors, seven measurements were performed by positioning the sample at each measurement. To check geometry errors, measurements were repeated for the geometry Bragg Brentano and Parallel Beams. In order to verify the reproducibility of the method, the measurements were performed in two different laboratories and equipments. The results were statistically worked out and the quantification the type A errors that suggests that is a significant difference between the methods and orientation of grooves directions.
\end{abstract}

\section{Introduction}

According with ASTM E2480/2017, generally, "a single test specimen may be measured more than once and the results combined to produce a test result if the protocol or test method so specifies" and "the same combination of operator and apparatus is used to obtain every test result on every specimen". In this study four test specimens were measured to investigate the uncertainty sources of XRD residual stress measurements and different laboratories, operators and apparatus - this is the main motivation for this work.

Nowadays, reliability is essential to quantify the residual stresses according to loading rates and mechanical behavior of structural materials. The X-ray diffraction technique is one of the most sensitive techniques for small variations of the crystalline lattice, since the $\mathrm{X}$-ray beam interacts with the interplanar distance and one of the most used non destructive techniques to evaluate stress level in polycrystalline materials [1,2]. In each obtained scan, the chosen diffraction peak position has to be accurately determined, i.e. the $2 \theta$ value has to be measured from a very broad and sometimes irregularly shaped peak [3]. The obtained $2 \theta$ values are then plotted in a graphic versus d-spacing where the linear regression is performed in order to obtain the slope and intercept values to be used with material elastic constants for the stress calculation $[3,4]$. The accuracy of the XRD- $\sin ^{2} \psi$ residual stress measurement depends on the minimization of various measurement errors derive from several sources. Materials to be measured, machine limitation, methods of analyses, manpower and software measurement conditions are the main big uncertain sources $[3,4,5,6]$.

(c) (1) Content from this work may be used under the terms of the Creative Commons Attribution 3.0 license. Any further distribution of this work must maintain attribution to the author(s) and the title of the work, journal citation and DOI. Published under license by Materials Research Forum LLC. 


\section{Experimental}

Ishikawa diagram is an excellent tool for mapping the possible sources of uncertainties [7]. An Ishikawa diagram was developed for residual stress measurements by XRD (Fig. 1).

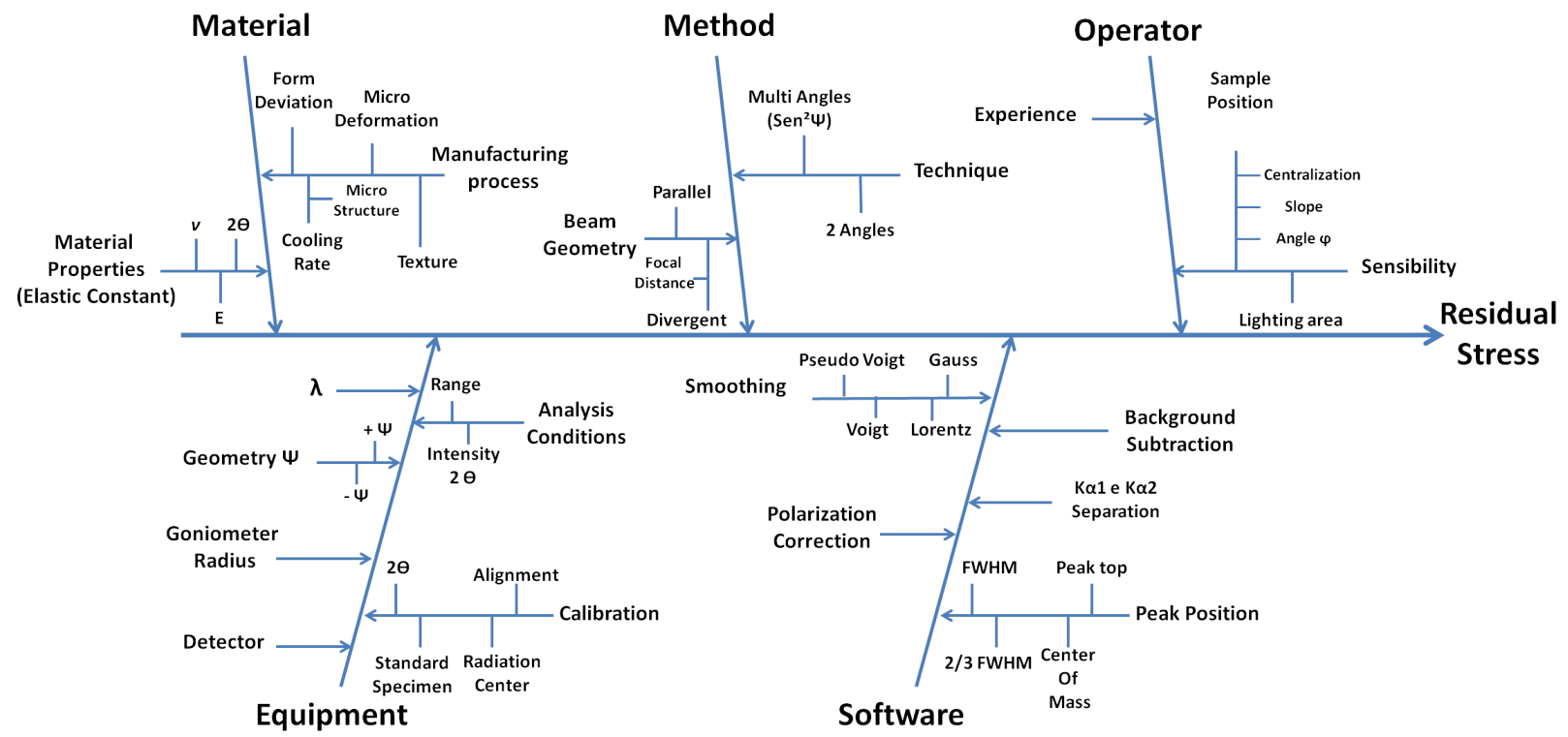

Figure 1 - Ishikawa diagram (Cause and Effect) to XRD Residual Stress Measurements.

Considering the sources of uncertainties grouped in the Ishikawa diagram the present work studies the influence of the material manufacturing process, the incident beam geometry, the positioning of the sample and with the collaboration of the laboratory located at the IPEN/USP it was possible to study the reproducibility of the measurements.

For this paper, two specimens were machined from a previously characterized drawn steel bar (AISI 1020). One of the specimens was polished with alumina suspension $(0.3 \mu \mathrm{m})$. The other specimen was grinded in 4 steps with $0.03 \mathrm{~mm}$ of grinding depth each, grinding wheel speed of $3500 \mathrm{rpm}$ and transverse motion of $0.10 \mathrm{~mm} / \mathrm{stroke}$.

As SAE HS784 and ASTM E915-10 standards recommend a ferritic steel powder with a grain size $>45 \mu \mathrm{m}$ and heat treated in a vacuum for relieving stress was used as reference.

The XRD residual stress measurements were made at UFRN in a Shimadzu model 7000 and repeated at IPEN in a Rigaku Ultima IV model, both with the target of CrK $\alpha$ (2.289 $\AA$ ). The XRD settings are shown in Table. 1.

Tabel 1 - XRD settings of the diffraction measurements for residual stress analysis.

\begin{tabular}{|c|c|c|}
\hline & UFRN & IPEN \\
\hline Method & Iso-inclination ( $\psi$-constant) & Iso-inclination( $\psi$-constant) \\
\hline Goniometer radius & $200 \mathrm{~cm}$ & $200 \mathrm{~cm}$ \\
\hline $2 \theta$ & $156^{\circ}(211)$ & $156^{\circ}(211)$ \\
\hline Range & $150^{\circ}$ to $162^{\circ}$ & $152^{\circ}$ to $161^{\circ}$ \\
\hline Step & $0.1^{\circ}$ & $0.1^{\circ}$ \\
\hline Counting time & 2 sec. & 2 sec. \\
\hline Speed & $3 \% \mathrm{~min}$. & $3^{\circ} / \mathrm{min}$. \\
\hline Current & $30 \mathrm{~mA}$ & $40 \mathrm{~mA}$ \\
\hline Voltage & $30 \mathrm{kV}$ & $40 \mathrm{kV}$ \\
\hline$\Psi$ Tilts & $\begin{array}{c}(0 ; 12.92 ; 18.43 ; 22.79 ; 26.56 ; 30 ; \\
33.21 ; 36.27 ; 39.23 ; 42.13 ; 45 .)\end{array}$ & $(-60 ;-48.59 ;-37.76 ;-25.65 ; 0)$. \\
\hline
\end{tabular}

For the analyzes performed in both laboratories a device that restricts the degrees of freedom of the sample positioning in the equipment was built, leading the specimens to be 
always positioned in the same place. To evaluate the influence of the positioning of the samples (analyzing the - method - error), 7 measurements were performed by repositioning the sample at each measurement and to evaluate the repeatability of the equipment, 7 uninterrupted measurements were performed without interfering with the positioning of the samples (analyzing the - equipment - error). In order to evaluate the influence of the machining direction, this same procedure was repeated in the grinded specimen for two conditions: aligning the $\mathrm{X}$-ray beam in the longitudinal direction of the grooves $(\varphi=0)$ and in the transversal direction of the grooves $(\varphi$ $=90$ ) of the specimen machined. For the polished sample and the iron powder a random position was initially established and then they were measured following the same procedures as cited above. The standards and technical books advise the use of parallel beam geometry for the calculation of residual stresses to minimize the effects of shape deviation, since the divergent beam geometry (Bragg Brentano) is sensitive to sample positioning and surface irregularities. For an evaluation of the beam geometry the analyzes were performed in both geometries: Pseudo-Parallel Beam and Divergent Beam.

The measurement uncertainty generally includes many variables which can be estimated based on the statistical distribution of the measurement series and they can be characterized by experimental standard deviations. The procedures used to treat the data obtained by the equipments were similar, but the software used by Shimadzu equipment was limited, it did not own some functions present in the software used by Rigaku equipment. The function adjustment for the smoothing and the intensity of the background radiation was determined by the measurement of 5 points on the left and right sides of each diffraction peak, adjusted by the absorption effect. Thereafter, the background radiation is subtracted, the diffraction line separations in $K \alpha 1$ and $K \alpha 2$ components were performed and thus the position of the $2 \Theta$ peak is obtained. After plotting the graph $\left(\varepsilon_{\varphi \psi}\right.$ versus $\left.\sin ^{2} \psi\right)$, a linear function is fitted in which the slope of this line multiplied by the elastic constant of the material gives the value of the residual stress.

\section{Results and discussion}

Initially, the average values and experimental standard deviations were calculated for the measurements performed at UFRN (Fig. 2) and IPEN (Fig. 3)
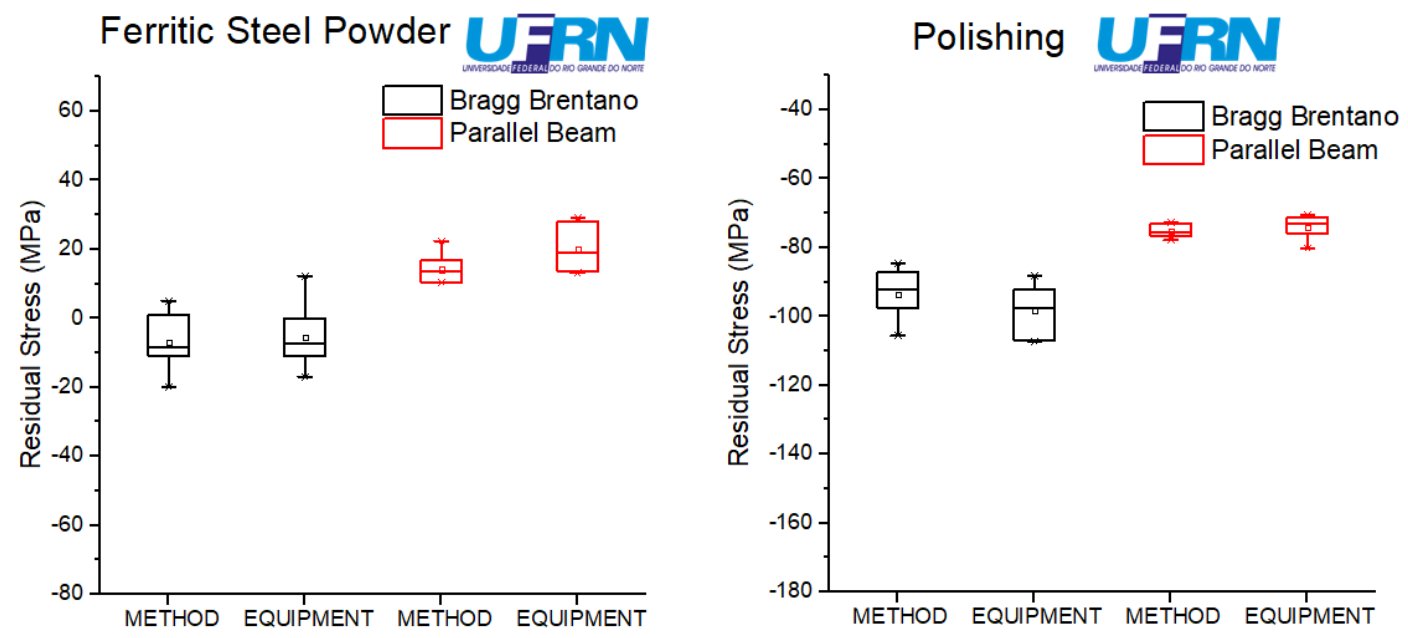

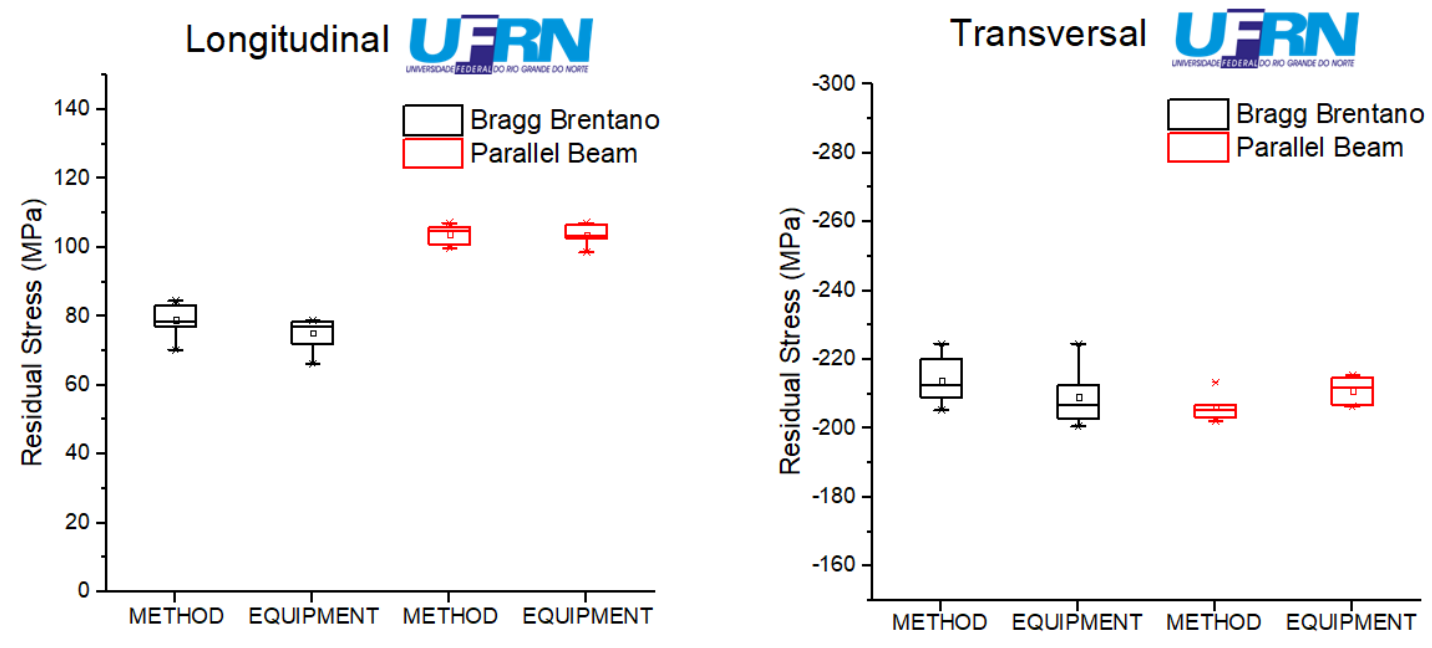

Figure 2 - Residual stress measurements obtained at UFRN.

This box chart graphic show four quadrants that represents $25 \%$ of the measurements and it's possible to use to study midhinge, range, mid-range, e trimean. The values obtained in the equipment from UFRN showed repeatability, demonstrated by the low values of experimental standard deviations. The values obtained with the iron powder were very close to zero (as expected according to standards and technical books) for both geometries. Although the values are within the acceptable range according to the standards (they can fluctuate between \pm 20 $\mathrm{MPa}$ ), in the geometry of divergent focusing (Bragg Brentano) it was possible to get closer to the true value. It was observed that, in most cases, the experimental standard deviation of the equipment showed to be slightly superior to the deviation of the measurement method, evidencing that the device built to perform the analyzes and the method adopted to carry out the measurements was not interfering in the quality of the presented results.

The measurements performed in the longitudinal direction to the grooves $(\varphi=0)$ presented to be tensile (positive values) while the measurements performed in the transversal direction to the grooves $(\varphi=90)$ presented to be compressive (negative values), indicating a heterogeneous state of stress, which is expected for grinding [8].
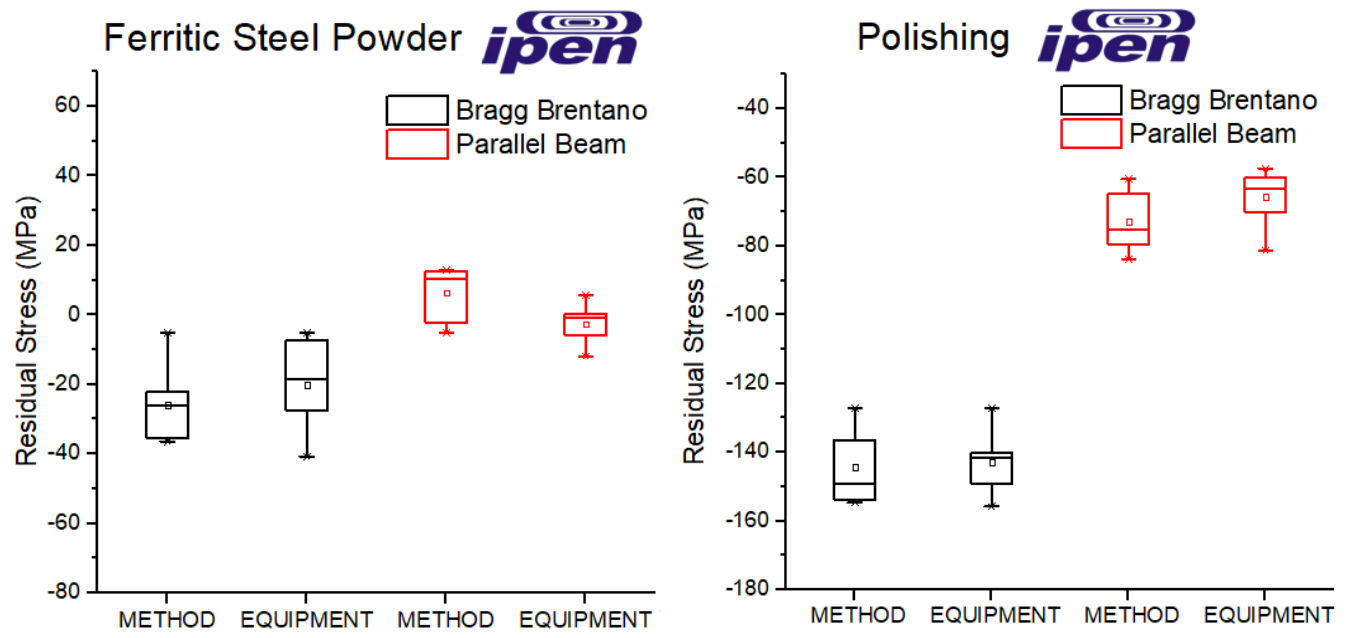

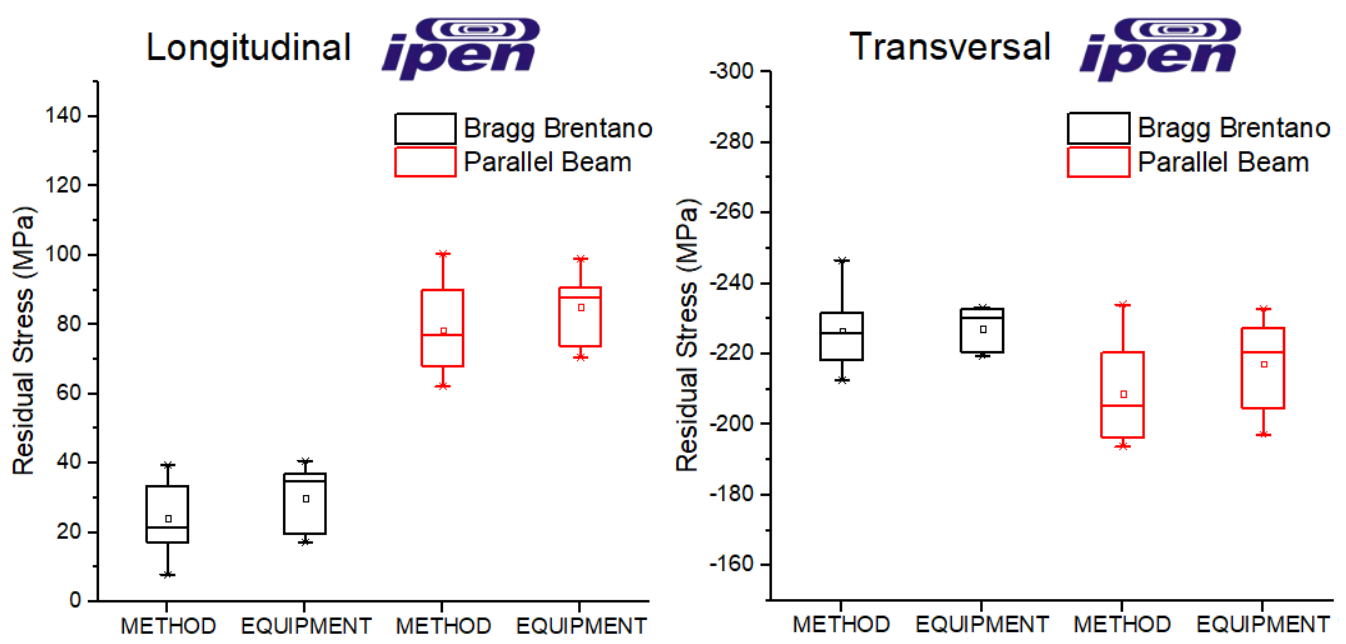

Figure 3 - Residual stress measurements obtained at IPEN.

Despite the low values of experimental standard deviations, the values obtained in the IPEN equipment were slightly higher than the values obtained in the UFRN and also demonstrated repeatability. The values of the iron powder were very close to zero for the geometries of pseudo-parallel beams, however for the geometry of divergent beams, the results were slightly above the acceptable by the standards. The aspect of the residual stresses measured in the IPEN equipment also showed to be consonant with those measured in the UFRN, but they diverged somewhat in module. The experimental deviations, analyzing the uncertainty of the method and the uncertainty of the equipment, proved to be very close, corroborating what was obtained with the UFRN equipment.

Given the measured data, using standard experimental deviations, type A (MPa) uncertainties were calculated from repeated observations, in this case, the uncertainty value (u) can be estimated as the standard deviation (s) divided by the number of the performed observations (Table 2).

Table 2 - Type A (MPa) uncertainty calculated from UFRN and IPEN measurements.

\begin{tabular}{c|c|c|c|c|c}
\hline & & \multicolumn{2}{|c|}{ Bragg Brentano } & \multicolumn{2}{c}{ Parallel Beam } \\
\hline & & Method & Equipment & Method & Equipment \\
\hline \multirow{2}{*}{ Ferritic Steel Powder } & UFRN & 3.07 & 3.53 & 3.80 & 1.52 \\
\cline { 2 - 6 } & IPEN & 4.01 & 4.60 & 2.80 & 2.11 \\
\hline \multirow{2}{*}{ Polishing } & UFRN & 2.65 & 2.73 & 0.74 & 1.26 \\
\cline { 2 - 6 } & IPEN & 3.95 & 3.35 & 3.26 & 8.01 \\
\hline $\begin{array}{c}\text { Grinded Specimen } \\
\text { (Longitudinal) }\end{array}$ & UFRN & 1.78 & 1.74 & 0.99 & 1.09 \\
\cline { 2 - 6 } $\begin{array}{c}\text { Grinded Specimen } \\
\text { (Transversal) }\end{array}$ & IPEN & 4.07 & 3.56 & 5.06 & 3.75 \\
\cline { 2 - 6 } & UFRN & 2.60 & 3.06 & 1.38 & 1.37 \\
\hline
\end{tabular}

The results showed that the equipment of the UFRN was more repetitive since the values of the uncertainty of type A suggested smaller values than for the measurements realized in the IPEN. Furthermore, most of the values for the geometry of divergent beams results were higher for both laboratories, indicating a greater susceptibility to the error, since there is a relation between the focal distance of the $\mathrm{X}$-ray source, the specimen positioning and the detector. Therefore, any variation in this distance causes shifting of the peak, leading to a fluctuation in the measurement. 


\section{Conclusions}

The results from this study suggest that the technique of X-ray diffraction using the geometry of pseudo-parallel beams is accurate and reproducible. The distinct responses between the geometry of pseudo-parallel beams and divergent beams proved to be different, but books and technical standards such as SAE HS784-03 consider that a difference of $\pm 20 \mathrm{MPa}$ within the uncertainty limit of the technique. The direction of the machining scratches plays an important role in the variation of residual stresses. The X-ray diffraction is considered as a reference technique for validation of other methods of residual stress analysis. However, the results of this study revealed that it is necessary to minimize the sources of uncertainties during the operation of Xray diffraction measurement, since this technique demonstrated considerable sensitivity to external influences, as demonstrated in the Ishikawa diagram. Hence, for a more detailed study on this quantification of this error, it is necessary to observe these variables inherent to the technique, constructing a worksheet of the measurement uncertainty estimation.

\section{References}

[1] P.J. Withers, H.K.D.H. Bhadeshia, Mater. Sci. Technol. 17 (2001) 355. https://doi.org/10.1179/026708301101509980

[2] B.D. Cullity, S.R. Stock, Elements of X-ray Diffraction, 3rd edition, Prentice Hall, Upper Saddle River, NJ, 2001, p. 435.

[3] M.E. Fitzpatrick, A.T. Fry, P. Holdway, F.A. Kandil, J. Shackleton, L. Suominen, Determination of Residual Stresses by X-ray Diffraction-Issue 2, DTI, 2005, Measurement Good Practice Guide No. 52.

[4] Q. Luo, A. H. Jones, High-precision determination of residual stress of polycrystalline coatings using optimised XRD-Sin ${ }^{2} \psi$ technique, Surface and Coatings Technology, 205(5)(2010)1403-1408. https://doi.org/10.1016/j.surfcoat.2010.07.108

[5] A T Fry, F A Kandil, A Study of Parameters Affecting the Quality of Residual Stress Measurements Using XRD, Materials Science Forum Vols. 404-407 (2002) pp. 579-584. https://doi.org/10.4028/www.scientific.net/MSF.404-407.579

[6] Czan, A., Zauskova, L., Sajgalik, M., \& Drbul, M. (2016). Triaxial Measurement Method for Analysis of Residual Stress after High Feed Milling by X-Ray Diffraction, Technological Engineering, 13(2), 31-33. https://doi.org/10.2478/teen-2016-0019

[7] L. Luca et al., "Study on Identification and Classification of Causes which Generate Welds Defects", Applied Mechanics and Materials, Vol. 657, pp. 256-260, 2014. https://doi.org/10.4028/www.scientific.net/AMM.657.256

[8] PINTO, Vinícius Carvalho. Influence of cutting parameters on the surface integrity of SAE 1045 steel after turning. 2017. 115p. Dissertation (Master in Mechanical Engineering) Technology Center, Federal University of Rio Grande do Norte, Natal, 2017. 